\title{
The Past Tense View of Counterfactuals Revisited
}

\author{
MORITZ SCHULZ \\ Universität Hamburg
}

\begin{abstract}
What is the best way of classifying different types of conditionals? According to what has become the traditional view, there is a substantive semantic difference between indicative conditionals and counterfactuals. Against this, it has been objected, primarily on grammatical grounds, that counterfactuals are merely past tense forms of indicative conditionals, expressing at a later time what the corresponding indicative conditional expressed at an earlier time. In this paper, I argue that closer inspection of the linguistic evidence shows that the case for the past tense view founders. The traditional view has it right after all.
\end{abstract}

$\prod$ HERE is a long-standing debate in philosophy about how the different types of conditionals are best classified. On the traditional picture, there are two basic kinds: indicative conditionals and counterfactuals (or subjunctive conditionals), which are taken to have a substantially different semantics. This view has been challenged by Dudman in a series of papers. ${ }^{1}$ Dudman claims that counterfactuals rather go with indicative 'will'-conditionals suggesting that they are simply a past tense form of these. Currently, the debate seems to be divided between the traditional view and the alternative picture promoted by Dudman. ${ }^{2}$ Although defenders of the traditional view have objected to Dudman's thesis, it remains unclear what exactly is wrong with Dudman's arguments. One reason seems to be that Dudman argues primarily on grammatical grounds, whereas the philosophical debate proceeds more by testing semantic intuitions with respect to various examples. The aim of this paper is to bridge this gap. It tries to give a defense of the traditional view by offering an explanation of what might be going on at the grammatical level. As it turns out, properly evaluating what we know

Contact: Moritz Schulz <moritz[dot]schulz[at]uni-hamburg[dot]de>

1. See Dudman (1983, 1984, 1988, 1991, 1992, 1994). Cf. also Gibbard (1981).

2. Opponents of Dudman's position include Bennett (2003) and Jackson (1987). Proponents are Strawson (1986) and Woods (1997). Adams (1975, ch. 4), Edgington (1995) and Weatherson (2001) go some way towards Dudman's position. See also Arregui (2009), Ippolito (2013) and Romero (2014) for linguistic approaches which share important similarities with Dudman. 
about the grammar of conditionals-not least through research in linguisticsactually supports the traditional view. But here is also a disclaimer: the paper does not attempt to supplement the traditional view with a semantics for indicatives and counterfactuals.

The structure of the paper is as follows. I will start with an explication of the view advocated by Dudman. Next I will look at three sources of evidence for Dudman's position. I will then present and discuss a counterexample to this view building on a similar example provided by Bennett (2003). With this in mind, I will then return to the three sources of evidence and try to explain how they are compatible with a denial of Dudman's view. Finally, I will extend the discussion to the relation between other types of conditionals.

\section{The Past Tense View}

It used to be a common assumption that there is a substantial semantic difference between indicative conditionals and counterfactuals, where it is often also presupposed that the two classes are themselves semantically homogenous and do not require finer subdivisions. But thanks to the work of Dudman and others, this view is no longer uncontested.

To explicate the rival account, we need to distinguish two classes of indicative conditionals based on their tense structure. Consider the following two conditionals:

(1) If they lose the match, they will fire the coach.

(2) If they lost the match, they fired the coach.

The first conditional has a present tense antecedent clause and a simple future formed in terms of the auxiliary modal 'will' in its consequent. Following Edgington (1995), let us refer to such conditionals as forward-looking indicative conditionals. The second conditional has simple past both in its antecedent and in its consequent. In a similar vein, we may call them backward-looking indicative conditionals.

Let us make the same kind of distinction for counterfactuals. Call counterfactuals of the form

(3) If they lost the match, they would fire the coach.

forward-looking counterfactuals. These counterfactuals employ simple past in their antecedent, but are nevertheless interpreted as being about the present or future. Their consequent is governed by the modal auxiliary 'would'. Similarly, let us call counterfactuals such as

(4) If they had lost the match, they would have fired the coach. 
backward-looking. ${ }^{3}$ They employ a double layer of past tense (pluperfect) in their antecedent and their consequent is again governed by 'would' with an added layer of past tense morphology. ${ }^{4}$

Now, it is an almost uncontested fact that a backward-looking counterfactual can be false while the corresponding backward-looking indicative conditional is true. The classical example which shows this is usually attributed to Adams (1970): 5

(5) If Oswald had not killed Kennedy, someone else would have.

(6) If Oswald did not kill Kennedy, someone else did.

Clearly, the indicative conditional is true (or at least acceptable), for we know that someone or other killed Kennedy. But the corresponding counterfactual seems to be false. As far as we know, Oswald was not part of a conspiracy. He acted on his own. So, if Oswald had not killed Kennedy, no one else would have.

Opponents of the traditional view of classifying conditionals usually accept the Oswald/Kennedy example as genuine (although they may disagree about the conclusions which should be drawn from it). But they typically hold that backward-looking counterfactuals are in fact like forward-looking indicative conditionals and differ from them only in temporal reference: backward-looking counterfactuals presently express what a forward-looking indicative conditional expressed at an earlier time. ${ }^{6}$ For instance, the suggestion is that the two conditionals

(7) If Oswald had not killed Kennedy, someone else would have.

(8) If Oswald does not kill Kennedy, someone else will.

are of the same basic kind. In this case, the counterfactual is taken to express now what the indicative conditional expressed a certain time before Kennedy's assassination. This view appears to have a better chance of escaping counterexamples of the Oswald/Kennedy type, for our judgement concerning the counterfactual coordinates quite well with our judgement concerning an utterance of 'If Oswald does not kill Kennedy, someone else will' made some time before Kennedy's assassination. Let us call the idea that forward-looking indicative conditionals

3. Backward-looking counterfactuals are usually interpreted as being about the past. But in certain contexts, they can also pertain to the present or future. See Ippolito (2003).

4. I use the term 'counterfactual' without any presumption as to the falsity of the antecedent. See Anderson (1951) and Stalnaker (1975, 145f.) for examples.

5. As a matter of fact, Adams (1970) gives the example with the consequents negated, with the aim of demonstrating that a counterfactual can be justified while the corresponding indicative conditional is not.

6. Cf. Dudman (1988, 1991). See also Strawson (1986) and Woods (1997, ch. 8). 
express at an earlier time what backward-looking counterfactuals express at a later time the past tense view of counterfactuals. ${ }^{7}$ Basically, the past tense view says that backward-looking counterfactuals and forward-looking indicative conditionals differ merely in temporal reference. Note that this is a semantic thesis: it is a thesis about which propositions are expressed by indicative conditionals and counterfactuals respectively across different contexts. The proposition expressed by a backward-looking counterfactual in a context $c_{1}$ is taken to be the same as the proposition expressed by a corresponding forward-looking indicative conditional in a suitably related past context $c_{2}$.

\section{Evidence from Morphology}

The past tense view gains support from considerations about the morphological make-up of counterfactuals. The antecedent of a counterfactual is usually formed from past tense morphology. The type of counterfactuals most relevant to the past tense view use past perfect in their antecedent. But simple past is also possible and if the main verb is 'to be', past subjunctive is allowed. These latter two possibilities are illustrated in the following examples:

(9) If the police officer followed her, Jackie would notice.

(10) If the police officer were smart, she would dress like a civilian.

Present tense or 'will' seems to be disallowed in the environment of counterfactuals. That past morphology seems mandatory in the antecedent of counterfactuals already goes some way toward establishing that they are past tense forms of indicative conditionals. ${ }^{8}$

Another important observation is that 'would' seems to be a past tense form of 'will' just like 'might' seems to be past tense of 'may', 'should' a past tense of 'shall' and 'could' a past tense of 'can' (see Dudman 1991; Iatridou 2000). ${ }^{9}$ It even seems that counterfactuals generally require a past tense modal to govern their consequent. ${ }^{10}$ For instance, counterfactuals formed in the following way all seem fine:

(11) If the police had followed her, Jackie would/might/could/should have noticed.

7. See Schulz $(2017,41-44)$ for a preliminary discussion. In the literature, the past tense view is sometimes referred to as the relocation thesis. See Bennett $(2003,13)$.

8. For more discussion of the role of past morphology in counterfactuals and a look at some cross-linguistic data, see Iatridou (2000). therein.

9. See also the discussion in Cariani and Santorio $(2018$, \$2.1) and the references given

10. Incidentally, this would also explain why there are no 'must'-counterfactuals, for 'must' does not have a past tense. 
If these observations are on the right track, then the modal auxiliary governing the consequent of a 'would'-counterfactual is simply a past tense form of the modal governing the corresponding indicative 'will'-conditionals.

Combining the observations about antecedent and consequent, it seems that the morphology used in counterfactuals is always past tense morphology. More precisely, the verb phrases used in counterfactuals are past tense forms of the verb phrases used in corresponding indicative 'will'-conditionals.

\section{Evidence from Indirect Speech}

There is potential evidence for the past tense hypothesis stemming from indirect speech reports. As Dudman $(1983,1992)$ has argued, in reporting what other people have said in the past, we seem to shift the tense of the reported utterance one step backwards. He then observes that future tense 'will'-utterances are reported using 'would'. This reinforces the idea that 'would' is a past tense form of 'will'.

Let us start with ordinary present tense utterances. In retrospect, we report such utterances by shifting the main verb of the reported speech into past tense:

(12) Jackie (in the past): 'I see a helicopter flying over the building.'

(13) Jackie said that she saw a helicopter flying over the building.

Depending on the context, there can be another option, however. If we take Jackie's assertion to still be valid, that is, if we assume that Jackie would hold that the helicopter still is flying over the building, we can also report her utterance by using present tense (cf. Comrie 1985, §5.3):

(14) Jackie said that she sees a helicopter flying over the building.

Let us turn now to past tense utterances. We report a past utterance of a past tense sentence using past perfect:

(15) Jackie (in the past): 'The police went away.'

(16) Jackie said that the police had gone away.

Thus, in reporting past utterances, we generally seem to add a layer of past tense to the sentence which was used. In a nutshell, this suggests the following hypothesis: the reporting clause expresses in the context of the reporter what the reported clause expressed in the context of the reportee. Call this the simple indirect speech hypothesis.

Interestingly, if the reported utterance uses 'will', the reporting utterance usually shifts to 'would': 
(17) Jackie (in the past): 'It will be a good time to rob the bank.'

(18) Jackie said that it would be a good time to rob the bank.

If the simple indirect speech hypothesis is correct, then 'would' must be a past tense of 'will', reinforcing the morphological hypothesis described in the previous section.

However, in full generality, the simple indirect speech hypothesis seems to be false. As Comrie $(1985, \$ 5.3)$ shows, we report predicted utterances by leaving the tense elements of the predicted utterance unaltered:

(19) Jackie says next Monday: 'It is a good day to rob the bank.'

(20) Jackie will say next Monday that it is a good day to rob the bank.

We cannot report the predicted speech by saying:

(21) Jackie will say next Monday that it will be a good day to rob the bank.

Such a report is taken to mean that next Monday Jackie will utter something like 'It will be a good day to rob the bank'. So, the simple hypothesis cannot be wholly correct.

These considerations only show that a general principle backing up the argument from indirect speech does not hold unrestrictedly. This does not mean that the targeted conclusion is not true: 'would' might still be a past tense of 'will'. The morphological evidence seems to be strong enough to establish this relation on its own. Comrie $(1985,75)$ also suggests that a better argument for the past tense view of 'would' can be given by considering past tense narrative constructions like the following:

(22) John left for the front; he would never return.

The occurrence of 'would' here is naturally interpreted as expressing that John does not return at any point later than the point at which he leaves for the front. So, 'would' expresses from a past perspective what 'will' would be taken to express if it were uttered at the relevant past point in time.

\section{Evidence from Sequences}

A possible source of evidence for the past tense view comes from the fact that we often make natural transitions between forward-looking indicative conditionals and corresponding counterfactuals after a certain amount of time has passed: 
I say 'If you touch that socket you will get an electric shock'. You don't touch it. I use my circuit-testing instrument, to show you: 'You see, I was right-if you had touched it, you would have got a shock'. Or, if the result is different, 'Funny, the power must be off. I was wrong. You wouldn't have got a shock if you had touched it'. (Edgington, 1995, 311)

Here Edgington describes a case which suggests that we evaluate a past utterance of a forward-looking indicative conditional by deliberating on a present utterance of the corresponding counterfactual. ${ }^{11,12}$

If sequences of an earlier utterance of a forward-looking indicative conditional and a later utterance of the corresponding counterfactual were always coassertable or, more generally, always demanded the same epistemic attitude, then this would be extremely strong evidence for the past tense view. For, if the past tense view fails, there will be an appropriate sequence in which the indicative conditional does not express the same proposition as the corresponding counterfactual. But then it should be possible to develop different epistemic attitudes towards the two propositions. If so, the conditionals would not always be coassertable. For this reason, I regard the argument from sequences as perhaps the strongest possible argument for the past tense view.

\section{Against the Past Tense View}

The past tense view is supported by sequences of conditionals moving from indicative to counterfactual, by morphological evidence, and, to some extent, by indirect speech reports. Nevertheless, it seems to be false. In this section, I discuss what I take to be a particularly strong counterexample to it. The task of explaining how the past tense view can fail despite the evidence in its favor will be deferred to subsequent sections.

To assess the past tense view, let us tease out one of its main consequences. Quite generally, if a sentence $s_{1}$ differs from a sentence $s_{2}$ merely in tense and the tensed elements in $s_{1}$ refer in a context $C_{1}$ to a time $t$ and the tensed elements in $s_{2}$ refer in a context $C_{2}$ to $t$ as well, then an epistemic subject who is aware of the passage of time should have the same epistemic attitude towards $s_{1}$ in $C_{1}$ as she has towards $s_{2}$ in $C_{2}$ unless she gains or loses relevant information while transiting from $C_{1}$ to $C_{2} \cdot{ }^{13}$ Call this the continuity principle. ${ }^{14}$ For instance, if I am

11. Although Edgington thinks that the difference between indicative conditionals and counterfactuals is '(mainly) one of tense' $(1995,314)$, she also thinks that the main line of divide remains the traditional one.

12. See also Weatherson (2001), who adopts a similar example from Jackson (1987).

13. I assume that the sentences are not context-sensitive in other respects.

14. It is developed further in Schulz (2010). 
aware of the passage of time and neither gain nor lose any relevant information, I should think today about 'Today is my birthday' what I thought yesterday about 'Tomorrow is my birthday'. If our epistemic attitudes attach primarily to propositions, and the relevant pairs of indexical sentences are taken to express the same proposition in the two contexts respectively, then the continuity principle can be seen to follow from the more general fact that we should not change our beliefs unless we gain or lose relevant information.

Now, applying the continuity principle to the past tense view of counterfactuals, we find the following consequence: if an epistemic subject has a certain credence in a forward-looking indicative conditional at a certain time, she should have the same credence in the corresponding counterfactual at some later point in time if she is aware of the passage of time and neither receives nor loses any relevant information. We can use this consequence to test the past tense view. The evidence from sequences seems to suggest that this consequence is indeed borne out, but we shall now see that this is not generally the case.

Against the past tense view, consider the following case (as described in Schulz 2017, 43). ${ }^{15}$ Police officer Shaker comes home in the afternoon. He finds a note in the kitchen saying 'I will be back tonight'. Unfortunately, he cannot identify who has written this. It may have been his daughter Linda or his wife Elizabeth. Both are reliable informants. It is much more likely, though, that it was written by Linda, because Elizabeth told him that she would be going on a business trip and would not be back before the next day. But Elizabeth's plans may have changed on short notice. In any case, Shaker seems right in thinking:

(23) If Linda is not home tonight, Elizabeth will be.

After this, Shaker leaves his house and spends the whole night in his office working on a case. What should Shaker think at the dawn of the new day about the following counterfactual?

(24) If Linda had not been home last night, Elizabeth would have been.

Shaker has to consider two possibilities. Consider first the most likely case that Linda wrote the note. Then the counterfactual can be assumed to be false: no one would have been home last night if Linda had not been, for Elizabeth would still have been on her business trip without any intentions of coming home early. In the second scenario, where Elizabeth wrote the note, we could assume the counterfactual to be true. In this case, Elizabeth was home and Linda was not, and even if she was, Elizabeth would still have been home if Linda had not been,

15. Bennett $(2003,345 f$.) discusses a similar example, which is a little more complex. Bennett's discussion does not control for the fact that no relevant information is acquired in the meantime. In Bennett's case, the subjects find out that the antecedent is false before the counterfactual is evaluated. 
for their plans did not depend on each other. Note that by assuming that the counterfactual is true in this case, we make life harder for ourselves, for the truth of the counterfactual is what the past tense view predicts. Now, given that the first case in which the counterfactual is false is much more likely than the second case (we can actually make it as likely as we wish), Shaker should think that the counterfactual is probably false. ${ }^{16}$ Yet he took the indicative conditional to be highly likely or even certain. He does not seem to have received any relevant information. If the two conditionals differed merely in temporal reference, Shaker's different epistemic attitudes would not be rational. But they seem to be.

The counterexample seems to arise because there is merely an evidential link between the antecedent and the consequent of the indicative conditional. Since Shaker knows that the note was written by either his daughter or his wife and he has no reason to distrust its content, he can conclude that if Linda is not home tonight, Elizabeth will be. But the information does not support a counterfactual connection between antecedent and consequent. As far as he knows, if Linda wrote the note, Elizabeth has no intentions of coming home that night and her plans are independent of Linda's whereabouts. Hence, later on, he has not much reason to think that if Linda had not been home, Elizabeth would have been.

In principle, one could try and see whether this diagnosis can be used to modify the past tense view in some way or other to make it more plausible. Merely excluding indicative conditionals which are solely accepted on the basis of an evidential link would deprive the view of much of its interest, though, for it would no longer constitute a classification of conditionals based on grammatical considerations. It would also run the risk of trivializing the proposal: that an indicative conditional which is accepted on the basis of a non-evidential link between antecedent and consequent can go counterfactual is a much weaker claim, a claim anyone might find acceptable having spelled out in some way what a non-evidential link amounts to.

Still, let me flag that the falsity of the past tense view is compatible with a more refined explanation of the differences between indicatives and counterfactuals in terms of aspect and tense only. Depending on whether the traditional view amounts to more than a mere denial of the past tense view, this need, however, not necessarily be in conflict with the traditional picture.

The Shaker case seems to show that the past tense view of counterfactuals is incorrect, and once the structure of the counterexample is realized, it is not

16. The probabilistic nature is essential to the example. If we try to make the first case certain, i.e., make it certain that Linda wrote the note, the indicative conditional could no longer be felicitously asserted in the original context, for it would have an antecedent which is certainly false. Despite being infelicitous, it may still be true, though, which would be all we need. 
hard to find more cases like it. The only thing needed are situations in which there is only an evidential link between antecedent and consequent, so that the indicative conditional is supported but the counterfactual can be undermined. What we have yet to see, however, is how a denial of the past tense view can be brought in line with the evidence in its favor.

\section{Time Shift and Morphology}

The evidence for the past tense view coming from morphological considerations is quite strong. The challenge is to explain how the relevant pairs of indicative conditionals and counterfactuals may differ merely in tense, and yet still differ semantically in more than just temporal reference. The counterexample to the past tense view suggests that there must be a real semantic difference, but it is not easy to point to any element in counterfactuals which might be responsible for that. In this section, we will look at possible ways of bringing the morphological evidence into harmony with a denial of the past tense view.

The interaction between tense and temporal reference in conditionals is quite intricate. As Dudman (1983, 1984) and Gibbard (1981) emphasize, the tenses in counterfactuals are not interpreted in the same way as they tend to be interpreted in unembedded environments. Rather, there is a systematic time shift in the interpretation of tense: past tense in counterfactuals is interpreted as being about present or future and past perfect is interpreted like simple past, and sometimes even like present or future tense (see Ippolito 2003).

To begin with, past perfect in the antecedent of counterfactuals does not usually receive a past perfect interpretation. Past perfect is typically used in freestanding sentences to refer to a point in time which lies earlier than a point in the past which is made salient either by the linguistic or the extra-linguistic context. However, in the antecedent of a counterfactual, past perfect does not receive this kind of doubly past interpretation. Rather, past perfect is typically interpreted like simple past or even as being about the present or the future:

(25) If Jackie had observed the bank more closely, she would not have chosen it as a target.

(26) If Paul had been with Jackie in the bank right now, he would have told her to give up.

(27) If Jackie had reached the flight to Paris tomorrow, she would have used her falsified French passport. (Context: she already got caught by the police.) ${ }^{17}$

17. The last two examples are modeled after similar examples by Dudman (1984, 149f.). 
In the first example, the past perfect merely takes us to a point or interval in the past with respect to which we make a counterfactual supposition. It does not take us to a point in the past which lies before some other point in the past. The subsequent two examples show that the forward time shift in the interpretation of past perfect is not limited to past interpretations; past perfect can also be interpreted as being about the present or future, although it seems that these interpretations are a little harder to access and need some overt marking ('right now') or a small dose of contextual stage setting. ${ }^{18}$ By default, the time shift seems to be only one step forward.

A similar phenomenon occurs in the interpretation of past tense in the antecedent of counterfactuals. It can receive a present tense interpretation, but it can also be interpreted as being about the future:

(28) If Fred knew about the job committee's decision, he would not prepare another application.

(29) If Juan came to the party, he would bring Jackie.

The first example is naturally interpreted as being about the present, whereas the second example concerns the future. It is striking that no clues are required to generate the future-oriented interpretation for the second example. Iatridou $(2000,250)$ persuasively suggests that this is not a coincidence but rather depends systematically on the Aktionsart of the verb phrase: a stative expression like 'know' is interpreted as present tense while an eventive verb like 'come' is taken to be future tense.

We may summarize the situation by saying that the interpretation of simple past in the antecedent of a counterfactual is shifted one step forward, where an extra layer is added by eventive verb phrases so that they are interpreted as concerning the future. (Eventive verb phrases do not have a similar effect in past perfect antecedents of counterfactuals.) The extra step triggered by eventive verb phrases can be overridden by overt temporal markers such as 'now' and should therefore only be regarded as a default.

The time shift phenomenon is a leak in the argument for the past tense view from morphological evidence. As we have seen, there is evidence that 'would' is the past tense of 'will'. From this it then follows that the consequent of a counterfactual such as 'Someone else would have killed Kennedy if Oswald had not done it' is a past tense form of the consequent of the corresponding forwardlooking indicative conditional, namely 'Someone else will kill Kennedy if Oswald does not do it'. We would still need to decide whether 'would' as it is used in counterfactuals really functions semantically as the past tense of 'will', but

18. See Arregui (2007) on the problem of what distinguishes the use of simple past and past perfect in counterfactuals in which the same temporal reference is made. 
I will set aside this question for now. It is worth noticing that the sentence 'Someone would kill Kennedy' is also a past tense form of the corresponding 'will'-sentence. The situation is similar for the antecedent of the two conditionals. The counterfactual's antecedent is the past perfect of the indicative conditional's antecedent. It does not employ its simple past, which would be 'Oswald did not kill Kennedy'. This puts an alternative hypothesis in place: the counterfactual 'If Oswald did not kill Kennedy, someone else would' is syntactically the simple past of 'If Oswald does not kill Kennedy, someone else will'. The counterfactual targeted by proponents of the past tense view is also a past tense form of the indicative conditional, but it contains an extra layer of tense.

The simple past counterfactual is not a candidate for underwriting the past tense view because it makes the same temporal reference as the corresponding indicative conditional. The two conditionals

(30) If Oswald does not kill Kennedy, someone else will.

(31) If Oswald did not kill Kennedy, someone else would.

typically talk about the same temporal regions when uttered at the same time because, as we saw before, the interpretations of the tenses in the counterfactual are shifted forward. Consequently, the counterfactual does not express on its standard use what the indicative conditional would express when uttered at an earlier time.

We get the right kind of difference in temporal reference for the pair of conditionals which proponents of the past tense view put forward:

(32) Earlier: If Oswald does not kill Kennedy, someone else will.

(33) Later: If Oswald had not killed Kennedy, someone else would have.

Forward-looking indicative conditionals differ from past perfect counterfactuals by two layers of tense. But they differ only by one layer as far as the temporal reference is concerned because the interpretation of tense in counterfactuals is shifted one step forward. There is then one layer of tense which does not make a contribution towards the temporal reference. This may put us in a position to explain how the past tense view can fail without denying the morphological evidence for it.

By default, grammatical tense and temporal reference are linked. Shifting only the tense back normally results in nothing more than shifting back the point of temporal reference. The resulting sentence will then express the same proposition as the original sentence at a different time. This rule is not without exceptions, though. As we have just seen, the simple past counterfactual can make the same temporal reference as the corresponding forward-looking indicative conditional. Now, in the case of a past perfect counterfactual, the simple link 
between tense and temporal reference is broken as well, albeit in a different way. Although we do find that the points of temporal reference of the counterfactual are shifted back one step and agree with the earlier utterance of the indicative conditional, this is not achieved by shifting back the tenses one step. The tenses in the counterfactual are shifted back two steps and so the counterfactual contains an extra layer of tense over and above the simple past. But then we can no longer conclude that the two conditionals express the same proposition, for the extra layer of tense may do extra work, so that the two conditionals differ in more than temporal reference. The extra layer of tense in counterfactuals which does not receive a temporal interpretation might be responsible for a semantic difference between indicative conditionals and counterfactuals. How could that be?

One idea would be that the tenses may have an underlying meaning which receives a temporal interpretation only by fixing a further parameter. ${ }^{19}$ The default setting of the hidden parameter could be assumed to trigger the temporal interpretation of the tenses. But in certain environments like the antecedent of a counterfactual, changing this parameter may allow for the specific interpretation of the tenses in counterfactuals.

Let me briefly sketch how this idea might be spelled out. In the temporal case, the past tense can be described as changing the time of evaluation along a temporal dimension. On a somewhat vague metaphorical level, one could extract from this the concept of a distance..$^{20}$ Distances can be measured on multiple scales. When the tenses receive their temporal interpretation, the underlying scale would be assumed to be the order of time. The idea could then be that in the environment of counterfactuals, the tenses can also receive a non-temporal interpretation which contributes to the specific meaning counterfactuals have. A possible option would be that the underlying scale of distance would then be metaphysical in nature introducing a certain order of possible worlds.

Such a story still leaves many questions open, in particular, whether the achieved effect is indeed semantic or merely pragmatic. ${ }^{21}$ Supporters of the idea that there is a profound semantic difference between indicative conditionals and counterfactuals could (partly) locate the source of this difference in the non-

19. Cf. Iatridou (2000). Dudman (1984) holds a similar but different view. He assumes that the layer of tense which does not contribute to the temporal location of the relevant event is used to mark a cut-off point after which possible histories pertaining to the truth of the counterfactual are allowed to depart from the actual history. However, he holds this also for the relevant indicative conditionals. See also the proposals in Ippolito (2003) and Khoo (2015).

20. For discussion, see Iatridou (2000, 245ff.) and the references given therein. She prefers the concept of exclusion over the one of distance. See also Schulz (2014) for an approach along similar lines. For some criticism, see Mackay (2015).

21. Iatridou (2000) takes the effect to be pragmatic, but she also takes the tenses to provide pragmatic constraints only.

Ergo • vol. 6, no. $41 \cdot 2020$ 
temporal interpretation of one layer of tense in counterfactuals. This would be one way of deriving the desired difference compatible with the morphological evidence and without postulating any ambiguity in the word ' if'. ${ }^{22}$

Unfortunately, we are not done yet. Dudman has argued in a variety of papers that the interpretation of tense in forward-looking indicative conditionals is similar to how the tenses are interpreted in counterfactuals. ${ }^{23}$ He claims that forward-looking indicative conditionals display the same time shift phenomenon as counterfactuals. If this were right, our current explanation of the semantic difference between the two kinds of conditional might be in danger, for the semantic contribution made by the extra layer of tense could be assumed to make the same contribution in indicative environments. In order to rebut this potential objection, let us take a closer look at the interpretation of tense in indicative environments.

First of all, note that the interpretation of past morphology in indicative conditionals is completely normal and does not involve any time shift. Simple past is interpreted in the environment of an indicative conditional in the same way as it is interpreted outside of it:

(34) If Jackie knew that there were surveillance cameras in the strongroom, she put on a mask.

(35) If Paul went to the party, he saw Jackie's new partner.

The interpretation of simple past follows the paradigm of interpreting it as being about the past. The same happens in the interpretation of past perfect. ${ }^{24}$

At first sight, it comes as a surprise, then, that indicative conditionals which employ present tense in their antecedent can involve a time shift:

(36) If Paul comes to the party, he will meet Jackie's new boyfriend.

The antecedent-clause is typically interpreted as being about the future.

A time shift of a present tense element is not quite as surprising as a time shift with respect to past morphology because present tense also allows for futureoriented interpretations in unembedded environments. For instance, we can felicitously say 'Paul comes to the party' when the party is tomorrow. It should be

22. There is an alternative explanation of the tenses in counterfactuals which would deliver a similar result. Rather than postulating two ways of interpreting the tenses-a temporal and a modal one-it is also possible to construe the extra layer of tense as a sign of a subjunctive use of the verb phrases. It is not without reason that counterfactuals are sometimes referred to as subjunctive conditionals. Cf. Edgington (1995, 240). For discussion, see Iatridou (2000, 265f.).

23. See, e.g., Dudman (1983, 1984, 1988, 1991, 1992, 1994). Cf. also Gibbard (1981).

24. As a result, a past perfect 'if'-clause like 'if Paul had gone to the party' is ambiguous between a proper past perfect interpretation and a shifted one, but this ambiguity will usually be resolved by the linguistic environment-indicative or counterfactual-in which the clause is embedded. 
noted, though, that the use of present tense for the purpose of future reference in unembedded environments is not unconstrained. ${ }^{25}$

As noted by Thomason and Gupta (1980), not all indicative conditionals with a present tense antecedent (and, for that matter, a 'will' governing the consequent) impose a time shift on the interpretation of the tensed elements:

(37) If Jackie is at the party, we will see her sooner or later.

Quite clearly, the antecedent concerns the present time and this is achieved without overt marking. Already at this point we can therefore draw the minimal conclusion that the time shift concerning present tense is not a general feature of indicative conditionals.

Iatridou (2000, 250f.) makes the striking observation that the interpretation of present tense indicative conditionals correlates with the interpretation of simple past for counterfactuals. We find the forward time shift in indicative conditionals when an eventive verb is employed. For stative verbs, no time shift occurs. This is parallel to the case of simple past in counterfactuals: the presence of an eventive verb phrase triggers as a default interpretation an extra step forward, so that simple past counterfactuals with an eventive verb phrase are usually interpreted by moving the reference time two steps forward, from past over present to future. In contrast, counterfactuals with a stative verb in simple past are interpreted as being about the present. It seems, then, that eventive verb phrases induce both in the environment of present tense antecedents of indicative conditionals and in the environment of simple past counterfactuals a forward time shift. ${ }^{26}$ Since counterfactuals generally involve a forward time shift, eventive verb phrases can add an extra layer. Now, given that present tense indicative antecedents with an eventive verb phrase are interpreted by moving the reference time only one step forward, from present to future, this suggests that the environment of indicative conditionals does not induce any kind of time shift by itself. This hypothesis is reinforced by the fact that indicative antecedents with past tense or with a stative verb phrase in present tense like (37) above do not involve a time shift at all.

What does this show about the relation between counterfactuals and indicative conditionals? The forward time shift we find in forward-looking indicative conditionals is not a general phenomenon and is triggered by the verb phrase employed, not by the conditional environment alone. Counterfactuals, on the

25. Comrie $(1985,47 \mathrm{f}$.) suggests that the relevant event needs to be presented as somehow scheduled.

26. One may wonder why eventive verbs can induce a forward time shift with respect to past tense in counterfactuals but only with respect to present tense in indicative conditionals. A possible explanation may be that the underlying feature of the interaction between eventive verbs and conditional environments only concerns the interpretation of present tense. It may occur in past tense counterfactuals because past tense here is interpreted like present tense due to the time shift already induced in virtue of the counterfactual environment alone. 
other hand, essentially bring about a forward time shift. In simple past counterfactuals, the presence of an eventive verb phrase adds an extra layer to a shift already induced by the counterfactual environment. For these reasons, we can continue to take the time shift in counterfactuals to be responsible for a semantic difference between the two kinds of conditional.

\section{Indirect Speech}

I return now to the question how 'will'-conditionals are reported in indirect speech. Somewhat surprisingly, it turns out that they do not seem to be reported in a way which would lend direct support to the past tense view.

To continue Edgington's circuit example, consider the forward-looking indicative conditional:

(38) Jackie (in the past): 'If you touch that socket, you will get an electric shock.'

Suppose first that you are still deliberating whether to touch the socket or not. We may then report Jacky's utterance by saying

(39) Jackie said (a moment ago) that if you touch that socket, you will get an electric shock. ${ }^{27}$

So, in such a case, we can simply repeat the earlier utterance of the indicative conditional. This parallels the case, mentioned earlier, of reports of present tense utterances which we still take to be valid.

If we do not take the conditional utterance to still be valid, the situation changes. So suppose you did not touch the socket and we focus on what Jackie said about this possibility. In this case we report (38) using

(40) Jackie said that if you touched that socket, you would get an electric shock.

So, in this case, we would indeed use past tense forms of antecedent and consequent to report the past utterance of a forward-looking indicative conditional. To be precise, the tenses in antecedent and consequent are rolled back exactly one step in accordance with what one might expect even on a restricted version of the simple indirect speech hypothesis.

But note that the embedded conditional is not the one to which the past tense view typically applies, for that would be the backward-looking counterfactual

(41) If you had touched that socket, you would have got an electric shock.

27. Intuitions do not seem entirely clear about whether to use 'will' or 'would' in the consequent. 
This is the one held to presently express what the indicative 'will'-conditional expressed at a past time (see section 1). But what we tend to use in indirect speech is actually the forward-looking counterfactual. Although reporting what Jackie said by using

(42) Jackie said that if you had touched that socket, you would have got an electric shock.

is not unacceptable, it seems appropriate to a lesser degree. ${ }^{28}$ It is more naturally construed as reporting an utterance by Jackie which concerned what was past relative to the time of Jackie's utterance than something which still lay in the future. So, on closer inspection, the evidence from indirect speech seems to conflict with the past tense view of conditionals, or at least it does not seem to support it in any straightforward way.

It is an interesting question as to why forward-looking indicative conditionals are reported in this way. It seems that in indirect speech contexts, the embedded counterfactuals do not display the time shift phenomenon they show in unembedded contexts. The antecedent is shifted back only one step in (40) to indicate that the reported present tense antecedent need no longer be valid and zero steps if it is still valid, as in (39). This could be taken to suggest that counterfactuals in indirect speech contexts have an interpretation in which they are not "real" counterfactuals, for their tenses do not shift. Given the absence of a time shift, they may indeed be interpreted as past tense forms of the indicative conditionals they are used to report.

\section{Evidence and Sequences}

Even if the past tense view fails, an explanation seems to be needed for why we often move freely from a forward-looking indicative conditional to a corresponding counterfactual after some time has passed, as described by Edgington in the quotation above $\left(\S_{4}\right)$.

We may start by observing that the case described by Edgington contains the information that the epistemic subject, after having asserted the forward-looking indicative conditional, finds out that the antecedent is false: you do not touch the socket and nothing stands in the way of this becoming common ground from there on. This makes it pragmatically impossible to use an indicative conditional. At least for a wide range of cases, it is commonly agreed that indicative conditionals are not felicitous if it is common ground between hearer and speaker that the antecedent is false. Under normal conditions, first asserting $A$ and then

28. As an anonymous referee disagrees, there is reason to investigate this verdict further. 
following-up on this with 'If $\neg A, B^{\prime}$ ' does not seem to be felicitous. ${ }^{29,30}$ Given that asserting an indicative conditional is no longer an option after we find out that the antecedent is false, we may have to choose a counterfactual form as our best means of conveying the content (or something close to it) of our original utterance.

The present conjecture seems to be confirmed by the fact that when the information about the falsity of the antecedent is taken away, it is more natural to use the backward-looking indicative conditional instead of the counterfactual. Let us redescribe Edgington's case in this way: I say 'If you touch this socket, you will get an electric shock'. I leave the room without having seen whether you touched the socket or not. Later on, I think: If she touched the socket, she got an electric shock. We would not normally think: If she had touched the socket, she would have got an electric shock. When we do not know the truth-value of the antecedent, it seems much more natural to use the backward-looking indicative conditional to reinforce what we expressed earlier by using the forward-looking indicative conditional. Thus, when the application conditions for the continuity principle are satisfied, the evidence from sequences actually supports a different conclusion: backward-looking indicative conditionals rather than backward-looking counterfactuals express at a later time what a forward-looking indicative conditional expressed at an earlier time. This is also in line with the Shaker example. At the next morning, Shaker is still justified in thinking: If Linda was not home last night, Elizabeth was. (We will come back to this thought in \$9.)

So far, the suggestion would be that we switch for pragmatic reasons to the backward-looking counterfactual in order to reinforce what we said in the past with a forward-looking indicative conditional. Using an indicative form is no longer a viable pragmatic option, so we use a counterfactual which tolerates it being common knowledge that the antecedent is false. Although I take this to constitute an essential part of a possible explanation of the problematic phenomenon, more has to be said, for if backward-looking counterfactuals are truthconditionally quite different from indicative conditionals, it remains unclear why

29. The explanation for this phenomenon is less clear. What to say will probably depend on which theory of conditionals one favors. On the material analyses, for instance, one can give a pragmatic explanation based on Gricean maxims, for the falsity of the antecedent would imply the corresponding conditional. For further discussion, see Stalnaker (1975) and von Fintel (1999).

30. As mentioned to me by Max Kölbel, a potential counterexample may be the use of conditionals in explanatory contexts: 'You won't touch it because if you do, you'll get a shock' (thanks to Max Kölbel). A potential response to this type of case would be to say that the embedded conditional is not intended to be evaluated in the context which results from updating on the antecedent-which here happens to be the explanandum-but rather in the original context in which the information that you won't touch the socket has not yet been established (one could equally well have said 'If you touch it, you'll get a shock and this is why you won't touch it'). 
they should be a suitable means, if only approximately, to convey what an indicative conditional expressed at a past point in time.

A possible thought would be that forward-looking indicative conditionals are usually about the future and knowledge of the future can often only be gained indirectly by combining knowledge about the present or the past with knowledge about causal dependencies. In the described situation, we can only know that you will get a shock if you touch the socket because we know that there is a causal link between touching an electrified object and getting an electric shock. Such causal connections will normally also support the corresponding counterfactual. For this reason, the truth of a forward-looking indicative conditional at an earlier time will usually support the truth of a corresponding counterfactual at a later time.

However, in situations in which there is merely an evidential link between antecedent and consequent, the corresponding counterfactuals may not be supported. What do we do in such cases? Somewhat surprisingly perhaps, it seems we still use the backward-looking counterfactual. Here is another case by Edgington which illustrates this:

There is a treasure hunt. The organizer tells me:

(43) I'll give you a hint: it's either in the attic or the garden.

Trusting the speaker, I think

(44) If it's not in the attic, it's in the garden.

We are competing in pairs: I go to the attic and tip off my partner to search the garden. I discover the treasure. 'Why did you tell me to go to the garden?' she asks.

(45) Because if it hadn't been in the attic it would have been in the garden: that's what I was told. (Or more pedantically: 'that's what I inferred from what I was told')

This doesn't sound wrong in the context. (Edgington, 2007, 212)

We may note that by finding the treasure in the attic, the antecedent of the conditional has turned out to be false. As before, if this information is taken away, a past indicative conditional is more appropriate. To see this, suppose the game is called off before anyone finds the treasure. I can then say 'If it was not in the attic, it was in the garden' ${ }^{31}$ On the other hand, using the counterfactual

31. It may be observed that the indicative conditional in this example of Edgington's does not employ 'will' in its consequent. As far as I can see, the case could be easily adjusted in this regard without losing its significance. 
would seem doubly wrong: pragmatically, because it would suggest that the antecedent is false, and semantically, because the counterfactual could easily be false if the organizers had no plans to put the treasure in the garden should they have decided against the attic.

A second observation is that the counterfactual used in this scenario, to repeat-

(46) If the treasure had not been in the attic, it would have been in the garden.

- quite clearly allows for a reading on which it is, or at least may very well be, false. This is the reading raised to salience already in the previous paragraph. The organizers' plans might have been such that if they had not put the treasure in the attic, they would have put it in the pool house, let's say. Then (46) would be false on a very natural reading despite the fact that it remains true that the treasure was either in the attic or in the garden.

As an intermediate conclusion, this suggests a somewhat more qualified picture. On their standard or primary reading, backward-looking counterfactuals do not express what a corresponding forward-looking indicative conditional expressed at an earlier time (as, for instance, witnessed by the Shaker case). However, counterfactuals may allow in certain contexts for a second reading on which they can be used to revive the indicative's content. The past tense view would continue to fail for the primary reading of counterfactuals, but could be upheld for a second, less visible reading that counterfactuals sometimes allow for.

It remains an open question whether this second reading of counterfactuals is a semantic or a pragmatic phenomenon. Edgington $(1995,2007)$ and in particular Khoo (2015) take it to be a semantic. On its second reading, we would evaluate a backward-looking counterfactual by going back to a past epistemic state-usually the past epistemic state of the speaker-and checking for the truth of the consequent in the then still open antecedent-worlds.

Alternatively, one could see the strong contextual clues necessary to trigger the second reading as providing a salient linguistic context in which the counterfactual is evaluated as implicitly embedded. For certain past tense constructions of modals (e.g., 'might have'), Hacquard (2006, 158f.) has proposed to construe certain readings as stemming from an elided 'I thought', so that a modal construction like

(47) The land might have contained oil.

can (but need not) be understood as saying

(48) Ithought The land might have contained oil. 
Such a reading may be salient when someone bought the land in light of (misleading) evidence that it contains oil. ${ }^{32}$ Looking back, she might utter (47) to justify her purchase.

Applying such an approach to the problem at hand has some initial plausibility. For example, Edgington's case contains explicit markers of earlier thought and speech ('... that's what I was told ...').

However, it is not clear whether this approach yields ideal results. Direct application of Hacquard's strategy to backward-looking counterfactuals would construe (46) as conveying, in the given context, the same as

(49) Ithought If the treasure had not been in the attic, it would have been in the garden.

Although not unacceptable as a report of what was earlier thought in terms of the indicative conditional, a higher degree of acceptability is enjoyed by using the simple past counterfactual (this was observed in $\S_{7}$ ):

(50) I thought that if the treasure were not in the attic, it would be in the garden.

However, when unembedded, we cannot hear the counterfactual

(51) If the treasure were not in the attic, it would be in the garden.

as repeating the content of an earlier utterance of the indicative conditional, for it concerns the present or the future. Something would have to be said of why this is so-why can we hear (46) but not (51) as occurring in the context of an omitted 'I thought'?

A possible response would be to take the extra layer of tense present in (46) to generate the embedding context of 'I thought' rather than to be implicitly embedded in it. So, one layer of tense would take us to a past epistemic context determined by what I thought, while the remaining material would be evaluated within that context. This would give us a reading of (46) close to (50). Edgington $(1995,2007)$ and Khoo (2015) assume that this is a semantic mechanism. In light of the fact that these readings seem to require it to be common ground that the antecedent is false and are further marked for indirect speech, a viable alternative assumption would be that they are the pragmatically generated result of a charitable reconstruction.

32. See also Stephenson $(2007,506 \mathrm{ff}$.) for a somewhat similar proposal. Stephenson suggests that epistemic modals are sometimes evaluated in an implicit explanatory context which in turn brings into play an implicit belief attribution. 


\section{Two Applications}

Before closing, I would like to focus on two related issues. The first concerns the question of whether there is a semantic difference between forward-looking indicative conditionals and simple past counterfactuals which make the same temporal reference. The second concerns the relation between forward-looking and backward-looking indicative conditionals. If one thought that forward-looking conditionals functioned semantically like counterfactuals and also accepted that the Oswald/Kennedy example shows that counterfactuals differ semantically from backward-looking indicative conditionals, one would predict a semantic difference between forward-looking indicative conditionals and backward-looking ones which goes beyond a mere difference in temporal reference. Regarding the two questions, I will argue for 'yes' in the first case and for 'no' in the second: simple past counterfactuals differ semantically from forward-looking indicative conditionals, but the latter differ from backward-looking indicative conditionals merely in tense. The resulting picture restores what has been called the traditional view (Edgington 1995, 311): there is a substantive semantic difference between counterfactuals and indicative conditionals, but within each class, the conditionals differ merely in temporal reference.

\subsection{Application I}

Lewis $(1973,48)$ is inclined to think that a counterfactual pertaining to the future such as

(52) If our ground troops entered Laos next year, there would be trouble.

has the same truth conditions as the corresponding forward-looking indicative conditional, i.e.,

(53) If our ground troops enter Laos next year, there will be trouble.

The idea that the two conditionals have the same truth conditions is, of course, compatible with a pragmatic difference between the two. It seems, for instance, that the use of the counterfactual tends to indicate that we take it to be less likely that the antecedent is true.

To challenge the idea that counterfactuals about the future are always equivalent to the corresponding indicative conditional, let us go back to the Shakercase. Compare the following two conditionals which are assumed to be evaluated when Shaker finds the note, written by either Linda or Elizabeth, and saying 'I will be back tonight':

(54) If Linda is not home tonight, Elizabeth will be.

(55) If Linda were not home tonight, Elizabeth would be. 
Given that Elizabeth's whereabouts are completely independent of Linda's, it seems that the counterfactual is false in the case in which Linda wrote the note. Since this is the most likely case, the counterfactual is likely to be false. As before, the indicative conditional is perfectly acceptable. Thus, counterexamples to the past tense view can also be used to identify a semantic difference between counterfactuals and indicative conditionals pertaining to the future.

\subsection{Application II}

We already mentioned that the Shaker example suggests a fairly unexciting alternative to the past tense view of counterfactuals: backward-looking indicative conditionals instead of counterfactuals express at a later time what a forwardlooking indicative conditional expressed earlier. In the Shaker case, the corresponding backward-looking indicative conditional should be accepted at the later point in time. Although the counterfactual should only be assigned a low subjective probability, the backward-looking indicative conditional seems to enjoy the same epistemic status at the later point in time as the corresponding forward-looking indicative conditional did earlier. ${ }^{33}$ So, the next morning Shaker should accept

(58) If Linda was not home last night, Elizabeth was.

This lends some support to the traditional view that on a semantic level, the past tense of forward-looking indicative conditionals is expressed by the corresponding backward-looking indicative conditionals rather than by a corresponding counterfactual. Given that neither the forward-looking counterfactual at the earlier time nor the backward-looking counterfactual at the later time are acceptable, this also suggests that the two counterfactuals differ merely in temporal reference.

There is a potential counterargument to the present idea that the past tense of forward-looking indicative conditionals is simply given by the corresponding backward-looking indicative conditionals. The argument given by Dudman makes up a very short Analysis paper which we can quote here in full length:

(1) It is a key tenet of 'the traditional way' that Doesn't-will, as it might be that if Oswald doesn't shoot Kennedy someone else will, is logi-

33. Of course, before the relevant time has passed, one way of reinforcing

(56) If Linda is not home tonight, Elizabeth will be.

can simply be

(57) If Linda is not home (now), Elizabeth will be.

Thanks to an anonymous referee for pointing this out. 
cally indiscernible from Didn't-did, that if Oswald didn't shoot Kennedy someone else did, the former merely formulating about the future what the latter formulates about the past. (2) A tenet no less central to the tradition has that someone will shoot Kennedy is logically indiscernible from that someone shot Kennedy, the former merely formulating about the future what the latter does about the past. (3) Didn't-did follows from the proposition that someone shot Kennedy. (4) A conspirator espousing the not untenable view that Oswald will shoot Kennedy in a plot that includes no back-up killer obviously maintains that someone will shoot Kennedy but need not accept Doesn't-will. Q.E.D. (Dudman, 2000)

This argument is fallacious. The line of thought Dudman uses to show that the forward-looking indicative conditional

(59) If Oswald doesn't shoot Kennedy, someone else will

does not follow from

(6o) Someone will shoot Kennedy

can equally be used to show that

(61) If Oswald didn't shoot Kennedy, someone else did

does not follow from

(62) Someone shot Kennedy

contrary to what Dudman thinks. To see this, suppose our conspirator leaves the city for good before Oswald committed his murder. The following night, sitting in a remote hideout lacking any sources of public information, our conspirator can be assumed to have the same degree of belief in (62) as he had in (60) the day before. He will be very confident that Kennedy was shot on the very same grounds on which he thought the day before that someone would shoot Kennedy, namely on the grounds that Oswald would shoot Kennedy. But if it is plausible that our conspirator should not have accepted (59) the day before-as it may seem to be-then he should neither accept (61) now in his hideout. Since he takes it to be very likely that someone shot Kennedy solely because he takes it to be very likely that Oswald managed to shoot Kennedy, he should equally reject the thought that if Oswald didn't shoot Kennedy, someone else did. So, it seems that the two varieties of indicative conditionals stand and fall together. ${ }^{34}$ The traditional view seems right after all.

34. Note that Dudman's argument can be rejected independently of deciding whether the indicative conditionals do indeed follow from 'Someone will shoot Kennedy' and 'Someone 
Recently, Khoo (2015) has put forward considerations which can, to a certain extent, be seen as a challenge to the present picture. For Khoo, forward-looking indicative conditionals are systematically ambiguous between an epistemic and a metaphysical reading. By default, they are read epistemically. But in certain contexts at least, a second reading can be made salient. ${ }^{35}$ The reading to which I have so far appealed is what Khoo describes as the epistemic reading. For Khoo, the forward-looking conditional (59) is thus ambiguous. Roughly, it is true on the epistemic reading iff a salient body of information excludes all worlds at which Oswald does not shoot Kennedy and no one else does so either. On the other hand, (59) is true on the metaphysical reading iff at all presently still open futures in which Oswald does not shoot Kennedy, no one else decides to do so either. If we know that Kennedy will be shot but the world is objectively so that there is no back-up killer, (59) would, according to Khoo's proposal, be true on its epistemic reading yet false on its metaphysical interpretation.

Whether the traditional view holds with respect to the relation between past tense and forward-looking indicative conditional would, if Khoo's proposal is right, depend on which reading of the forward-looking indicative conditional we focus on. It would be right for the epistemic reading, but false for the metaphysical one.

Although I am open to Khoo's suggestion, let me mention a few reasons for concern. As a preliminary point, note that a univocal analysis of indicative conditionals is entirely compatible with them being accepted (or rejected) on different grounds. I may accept the conditional 'If Linda is not home tonight, Elizabeth will be' on purely evidential grounds, for instance because I know that one of the two will be home tonight. But I could equally well have accepted the conditional because I think that Linda is going to call Elizabeth if she doesn't make it home by tonight, in which case Elizabeth will decide to return early. Almost

shot Kennedy' respectively. This makes it much easier to counter Dudman's considerations, for deciding about this question would effectively require to decide whether the material analysis of indicative conditionals is correct. All that needs to be shown in order to restore the traditional view is that either both follow in this way or neither does. In this respect, the present reply is more neutral than the one offered by Bennett $(2003,354)$ who rejects the relevant implication, i.e., premise (3) of Dudman's argument.

35. On Khoo's account, there is a beautiful resolution of the past tense view available. On his view, counterfactuals, too, are ambiguous between an epistemic and a metaphysical reading, only that in the case of counterfactuals, the metaphysical reading is the default one. So, when comparing the default readings-metaphysical for counterfactuals but epistemic for indicative conditionals - the past tense view would fail, just as I have argued. However, the past tense view would have two true variants: it would be true for both metaphysical and epistemic readings of the two kinds of conditionals, i.e., if we pair metaphysical readings of counterfactuals with metaphysical readings of indicative conditionals and, mutatis mutandis, epistemic readings of counterfactuals with epistemic readings of indicative conditionals, the past tense view would in each case be validated. 
any proposition can be believed on different grounds. Hence, the possibility of accepting a sentence based on different grounds does nothing to suggest that it is ambiguous.

Moreover, we should expect that the context of conversation will often provide clues about the likely grounds a speaker has for her assertion. For example, when the tour manager of a band says 'If tonight's concert is cancelled, there will be a another concert tomorrow' she is likely taken to have causal (i.e., metaphysical) grounds for her assertion, including, for instance, information to the effect that the band has agreed to give a second concert if tonight's gig is cancelled. So, if indicative conditionals allow for a univocal (possibly epistemic) analysis, one can still recover what might seem like a metaphysical reading as a conversational implicature.

The question of whether forward-looking indicative conditionals have a second, metaphysical reading turns on whether one thinks the following remark has a semantically coherent interpretation:

(63) I know for sure that either Linda or Elizabeth will be home tonight. But I don't think that if Linda is not home tonight, Elizabeth will be.

To me, this remark seems incoherent (Khoo 2015 disagrees for analogous cases). That does not mean that one can't figure out what the speaker might be getting at. Yet it seems that if there is a coherent content which the speaker intends to express, she has chosen the wrong words with which to do so.

Another way of testing whether forward-looking indicative conditionals have a metaphysical interpretation is to see whether contingent a priori truths can be successfully denied in the consequent of such conditionals. If forward-looking indicative conditionals have a metaphysical interpretation, one would expect this to be so. We find this expectation borne out with respect to counterfactuals. Contingent a priori truths can, without much set-up, be denied in the consequent of counterfactuals:

(64) If my parents had never met, I would not have existed.

(65) If I had not caught the train, I would not be here now.

Contingent a priori truths concerning the future are somewhat rare. But consider the following examples. Let us stipulate 'Hiob' to name the next victim of a robbery in L.A. and suppose Harry and Frank are, as a matter of fact, the ones going to rob Hiob. Now consider the following two conditionals:

(66) If Paul stops drinking, he will be more successful than he actually will be.

(67) If today Harry and Frank give up robbing people, Hiob will not be the next victim of a robbery in L.A. 
As far as I can see, these conditionals do not have an acceptable reading, contrary to what one might have expected if they had a metaphysical interpretation. I admit, however, that this piece of evidence is somewhat marginal because not only does it rely on the availability of contingent a priori truths about the future, but it also needs to make certain assumptions about how they embed into conditional contexts.

\section{Conclusion}

According to the past tense view, counterfactuals merely express what a forwardlooking indicative conditional expressed at an earlier point in time. I have argued, without making any substantial semantic assumptions, that the past tense view is false. Not only are there direct counterexamples to it, but the evidence cited in its favor can be explained without adopting this view, in some cases even in a much better way.

\section{Acknowledgements}

An earlier version of this paper was presented at a research seminar in Barcelona 2013 and at a workshop in Berlin 2016. I would like to thank all the participants for their helpful comments. Special thanks are due to Max Kölbel and two anonymous referees of this journal. The paper profited from the generous support of the DFG-funded project "Knowledge and Decision" (SCHU 3080/3-1; PI: Moritz Schulz).

\section{References}

Adams, Ernest W. (1970). Subjunctive and Indicative Conditionals. Foundations of Language, 6(1), 89-94.

Adams, Ernest W. (1975). The Logic of Conditionals. Reidel.

Anderson, Alan R. (1951). A Note on Subjunctive and Counterfactual Conditionals. Analysis, 12(2), 35-38.

Arregui, Ana (2007). When Aspect Matters: The Case of Would-Conditionals. Natural Language Semantics, 15(3), 221-264.

Arregui, Ana (2009). On Similarity in Counterfactuals. Linguistics and Philosophy, 32(3), $245-278$.

Bennett, Jonathan (2003). A Philosophical Guide to Conditionals. Oxford University Press.

Cariani, Fabrizio and Paolo Santorio (2018). Will Done Better: Selection Semantics, Future Credence, and Indeterminacy. Mind, 127(505), 129-165. 
Comrie, Bernard (1985). Tense. Cambridge University Press.

Dudman, Victor H. (1983). Tense and Time in English Verb Clusters of the Primary Pattern. Australian Journal of Linguistics, 3(1), 25-44.

Dudman, Victor H. (1984). Conditional Interpretations of If-Sentences. Australian Journal of Linguistics, 4(2), 143-204.

Dudman, Victor H. (1988). Indicative and Subjunctive. Analysis, 48(3), 113-122.

Dudman, Victor H. (1991). Interpretations of "If"-Sentences. In Frank Jackson (Ed.), Conditionals (202-232). Clarendon Press.

Dudman, Victor H. (1992). A Popular Presumption Refuted. The Journal of Philosophy, 89(8), 431-442.

Dudman, Victor H. (1994). On Conditionals. The Journal of Philosophy, 91(3), 113128.

Dudman, Victor H. (2000). Classifying "Conditionals": The Traditional Way Is Wrong. Analysis, 6o(2), 147.

Edgington, Dorothy (1995). On Conditionals. Mind, 104(414), 235-329.

Edgington, Dorothy (2007). On Conditionals. In Dov M. Gabbay and Franz Guenthner (Eds.), Handbook of Philosophical Logic (Vol. 14, 127-221). Springer.

von Fintel, Kai (1999). The Presupposition of Subjunctive Conditionals. MIT Working Papers in Linguistics, 25, 29-44.

Gibbard, Alan (1981). Two Recent Theories of Conditionals. In William L. Harper, Robert Stalnaker, and Glenn Pearce (Eds.), Ifs (211-248). Reidel.

Hacquard, Valentine (2006). Aspects of Modality. Doctoral dissertation. MIT. Retrieved from https://dspace.mit.edu/handle/1721.1/37421

Iatridou, Sabine (2000). The Grammatical Ingredients of Counterfactuality. Linguistic Inquiry, 31(2), 231-270.

Ippolito, Michela (2003). Presuppositions and Implicatures in Counterfactuals. Natural Language Semantics, 11(2), 145-186.

Ippolito, Michela (2013). Subjunctive Conditionals. MIT Press.

Jackson, Frank (1987). Conditionals. Basil Blackwell.

Khoo, Justin (2015). On Indicative and Subjunctive Conditionals. Philosophers' Imprint, 15(32), 1-40.

Lewis, David (1973). Counterfactuals. Blackwell.

Mackay, John (2015). Actuality and Fake Tense in Conditionals. Semantics \& Pragmatics, 8(12), 1-12.

Romero, Maribel (2014). 'Fake Tense' in Counterfactuals: A Temporal Remoteness Approach. MIT Working Papers in Linguistics, 2, 47-63.

Schulz, Katrin (2014). Fake Tense in Conditional Sentences: A Modal Approach. Natural Language Semantics, 22(2), 117-144.

Schulz, Moritz (2010). The Dynamics of Indexical Belief. Erkenntnis, 72(3), 337351.

Schulz, Moritz (2017). Counterfactuals and Probability. Oxford University Press. 
Stalnaker, Robert (1975). Indicative Conditionals. Philosophia, 5(3), 269-286.

Stephenson, Tamina (2007). Judge Dependence, Epistemic Modals, and Predicates of Personal Taste. Linguistics and Philosophy, 3o(4), 487-525.

Strawson, Peter F. (1986). "If" and " $\supset$ ". In Richard E. Grandy and Richard Warner (Eds.), Philosophical Grounds of Rationality (229-242). Clarendon.

Thomason, Richmond H. and Anil Gupta (1980). A Theory of Conditionals in the Context of Branching Time. The Philosophical Review, 89(1) , 65-90.

Weatherson, Brian (2001). Indicatives and Subjunctives. Philosophical Quarterly, 51(203), 200-216.

Woods, Michael (1997). Conditionals. Oxford University Press. 